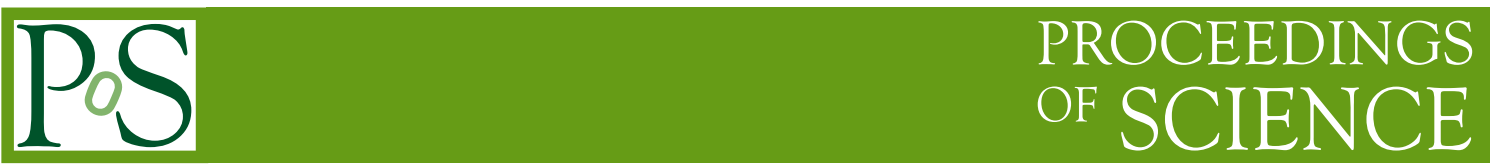

\title{
Results from the CUORE experiment
}

\author{
Antonio Branca* on behalf of the CUORE Collaboration \\ Università degli Studi di Padova and INFN-Sezione di Padova \\ E-mail: branca@pd.infn.it
}

The Cryogenic Underground Observatory for Rare Events (CUORE) is the first bolometric experiment searching for neutrinoless double beta decay $(0 v \beta \beta)$ that has been able to reach the one-ton scale. The detector consists of an array of $988 \mathrm{TeO}_{2}$ crystals arranged in a compact cylindrical structure of 19 towers. The construction of the experiment was completed in August 2016 with the installation of all towers in the cryostat. Following a cooldown, diagnostic, and optimization campaign, routine data-taking began in spring 2017. In this talk, we present the $0 v \beta \beta$ results of CUORE from examining a total $\mathrm{TeO}_{2}$ exposure of $86.3 \mathrm{~kg} \cdot y r$, characterized by an average energy resolution of $7.7 \mathrm{keV} \mathrm{FWHM}$ and a background in the region of interest of 0.014 counts $/(\mathrm{keV} \cdot \mathrm{kg} \cdot \mathrm{yr})$. In this physics run, CUORE placed the current best lower limit on the ${ }^{130} \mathrm{Te} 0 v \beta \beta$ half-life of $>1.3 \cdot 10^{25} y r$ (90\% C.L.). We then discuss the additional improvements in the detector performance achieved in 2018, the latest evaluation of the CUORE background budget, and we finally present the most precise measurement of the ${ }^{130} \mathrm{Te} 2 v \beta \beta$ half-life to date.

European Physical Society Conference on High Energy Physics - EPS-HEP2019 -

10-17 July, 2019

Ghent, Belgium

${ }^{*}$ Speaker. 


\section{The CUORE experiment}

CUORE $[1,2]$ is the first ton scale bolometric detector built with the primary goal to search for neutrinoless double beta decay $(0 v \beta \beta)$. Ideally this process has a clear signature: a monochromatic peak in the energy spectrum of the two electrons in the final state. Experimentally we must take care of the energy resolution and background contribution, as well as select an isotope candidate for $0 v \beta \beta$ decay with high natural isotopic abundance or enriched, ensure high detector efficiency and mass, guarantee good detector stability over a long period of time. All these parameters drive the experimental sensitivity.

CUORE exploits the bolometric technique [3, 4]: crystals of $\mathrm{TeO}_{2}$ are in thermal equilibrium, through a weak thermal coupling, with a bath at cryogenic temperature. A particle releasing its energy into the crystal produces a rise in temperature of the crystal itself, which is read out through a resistive thermometer (Neutron Transmutation Doped (NTD) thermistors) as a voltage variation. The bolometric technique ensures high detection efficiency and energy resolution. The chosen isotope ${ }^{130} \mathrm{Te}$ is a good candidate for $0 v \beta \beta$ given its high natural isotopic abundance of $34.2 \%$, higher than any other available isotope. Moreover it has a high Q-value for the process, $Q_{\beta \beta}=$ $(2527.515 \pm 0.013) \mathrm{keV}$, above almost all the environmental $\gamma$ background. Also, thanks to the scalability of the bolometric technique, detectors of large mass can be built.

Since CUORE is searching for a very rare event, it is extremely important to reduce as much as possible the background sources. For this reason, the experiment is located at the LNGS underground laboratories, at an average depth of about 3600 m.w.e., reducing natural radioactivity from outside the detector. Moreover, strict protocols have been developed and applied for crystals production and detector assembling, and new techniques have been tested and employed to clean the copper parts near crystals. These precautions allow to reduce natural radioactivity from the detector itself. Furthermore, suspensions and dumping systems, as well as noise cancelling tools [5], have been adopted to reduce the induced noise due to mechanical vibrations.

The cryogenic system [6] is a challenge by itself, able to cool down a large mass to cryogenic temperatures in few weeks and in a low radioactive environment. The system is a cryogen-free cryostat, allowing to achieve a high duty-cycle. A combination of a fast-cooling system with 5 pulse tubes (PTs) is employed to bring down the temperature at $4 \mathrm{~K}$. In the last stage of the cooldown a dilution unit (DU) allows to bring the temperature down to $10 \mathrm{mK}$, which is the temperature of operations. The use of pure copper and roman lead shielding allows to further reduce background from the cryostat itself.

\section{Commissioning and optimization phases}

The cryostat underwent a commissioning phase that lasted about two years and ended in March 2016: more than one run has been performed during this period, each time to check a different part of the system. In summary this important phase allowed to demonstrate that a base temperature as low as $6.3 \mathrm{mK}$ can be reached and kept stable for a long period of time. The electronics, DAQ and calibration system have been successfully tested. An array of 8 crystals was also installed to get a first raw estimation of the detector resolution, which turned out to be $10 \mathrm{keV}$ without any 
detector optimization. The outcome of the cryostat commissioning was that the experimental setup was ready for the installation of the actual detectors.

The assembling of the modules holding the crystals, called towers, and their installation on the cryostat followed a strict protocol developed during the assembling of CUORE-0 [7], the predecessor experiment of CUORE. CUORE-0 has been an important demonstrator to test installation procedures and material cleaning techniques, but it has also been an experiment producing physics results by itself $[8,9,10,11]$. The assembling of the CUORE towers has been performed within glove boxes in nitrogen atmosphere to avoid radioactive recontamination, and their installation in a protected area inside the clean room flushed with radon free air. All 19 towers have been installed between July-August 2016; the specifics of the detector design are: 988 crystals overall, for a total amount of $206 \mathrm{~kg}$ of ${ }^{130} \mathrm{Te}$; their arrangement is such that the crystals form closely packed arrays with high granularity, and the material employed in the tower holding structure is minimized to reduce the radioactive background from the material surrounding the bolometers. The goals are to reach a background as low as $10^{-2}$ counts $/(\mathrm{keV} \cdot \mathrm{kg} \cdot \mathrm{yr})$ [12] and an energy resolution in the region of interest of $5 \mathrm{keV}$ FWHM, values that lead to a projected sensitivity of $T_{1 / 2}^{0 v}>9 \cdot 10^{25} \mathrm{yr}$ in 5 years at $90 \%$ confidence level [13].

The cryostat has been closed during September-October 2016 and right after CUORE started with the detector cool-down on December 5th. The cool-down phase lasted 26 days overall, not taking into account two periods devoted to system debugging; the last 4 days the DU was switched on and the temperature reached the stable base temperature of $7 \mathrm{mK}$. At this point, detector optimization campaigns started and have been alternated with data-taking periods.

Temperature scans have been performed to optimize the detector resolution and the NTDs resistances around their design values: in the first scan a temperature of $15 \mathrm{mK}$ has been identified as the best working temperature for the data taking; the second scan was performed in between the two data-taking periods during 2017, to check the settings. Fixed the working temperature also the NTDs resistances are set: the correct bias current to be supplied to the thermistors are identified through a procedure developed for CUORE. For each bolometer the characteristic voltage versus current curve, Load Curve (LD), is measured; moreover, the amplitude of a reference pulse, the noise RMS and corresponding SNR are measured at each point of the LD curve. The bias current is chosen such that the SNR is maximized, and at the same time at values lower than the inversion point, to avoid distorted signal shapes and get the correct response from each bolometer.

Tools have been developed to minimize the vibrational noise induced by the PTs: an improvement of the temperature stability has been obtained by using linear drives to control the PTs rotating valves (see Fig. 1); a scan of the PTs phases has been introduced, allowing to find the configuration inducing the minimum vibrational noise into the detector [5].

The first phase of detector optimization was completed by the end of April 2017, when CUORE started taking the first data for physics analysis. The typical data-taking period consists of three/four weeks of data, bracketed by two calibration periods, one at the beginning and another at the end to check the initial calibration. We had two of these periods, called datasets, in 2017. In between a second phase of detector optimization took place, which consisted in different activities; for instance, it was introduced the PTs phase scan to refine the reduction of the noise. The effect of this second optimization phase is an improvement of the detector resolution, from $9 \mathrm{keV}$ to $7.4 \mathrm{keV}$ (see Fig. 2). This results demonstrates the importance of the detector optimization phases 


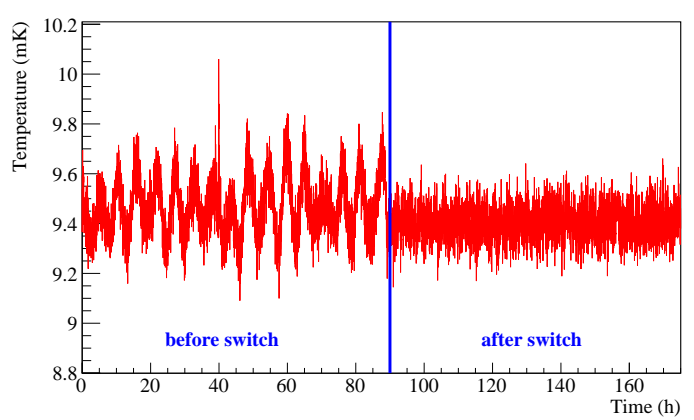

Figure 1: Base temperature at the mixing chamber plate of the CUORE cryostat, before (left) and after (right) the switch-on of the linear drives. The effect of the linear drives is an improvement of the temperature stability.

\section{for CUORE.}

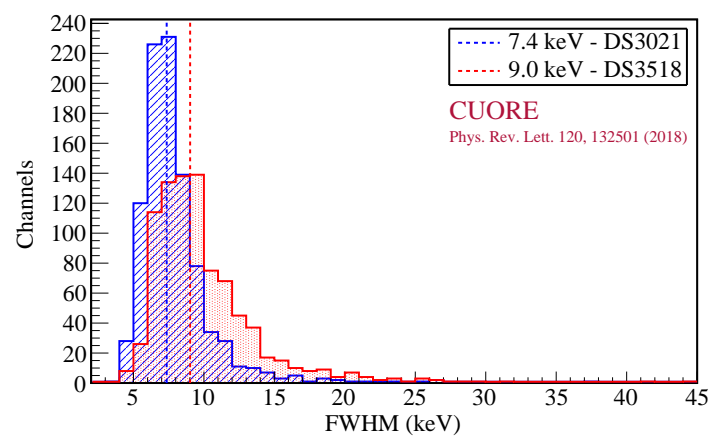

Figure 2: Distribution of the crystals resolution at the $2615 \mathrm{keV}^{208} \mathrm{Tl} \gamma$-line in calibration data. In red is the resolution from the first dataset, and in blue the one from the second dataset, after the detector optimization phase that took place in between the two data taking periods.

\section{First $0 v \beta \beta$ results}

The data acquired during 2017 have been analyzed to search for neutrinoless double beta decay [15]. The total exposure of $\mathrm{TeO}_{2}$ is $86.3 \mathrm{~kg} \cdot \mathrm{yr}$. A selection procedure is applied to discard background events. Noisy periods, due for instance to activities in the laboratory, are discarded (about $1 \%$ of the total exposure). Basic pulse quality requirements are applied to avoid spikes and pile-up. Since radioactive contaminants are likely to produce signals in more then one crystal in a given time window, an anti-coincidence selection is performed. A pulse shape analysis is carried out to select only signal events for which the waveform is consistent with that of a true signal. To do this 6 variables sensitive to the pulse shape are used to build a distance in the parameter space from the mean value of a typical signal. A threshold maximazing the experimental sensitivity is obtained on this distance. The total selection efficiencies are $(75.7 \pm 3) \%$ for the first dataset and $(83.0 \pm 2.6) \%$ for the second dataset. 


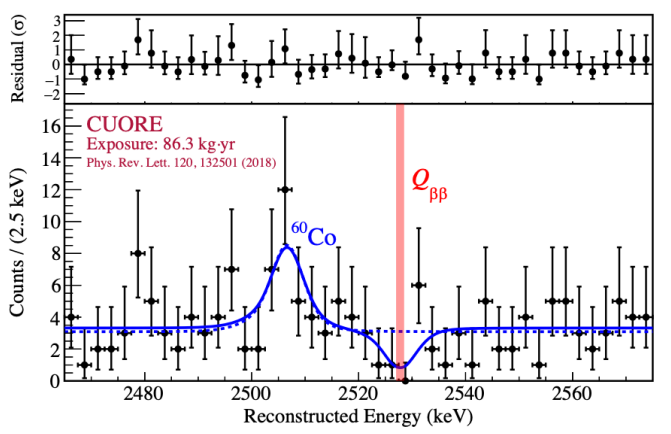

Figure 3: Bottom: UELM fit (blue solid line) to the energy spectrum in the ROI; the dashed blue line shows the fit for the zero signal hypothesis; the verical red band corresponds to the $\mathrm{Q}$-value of the $0 v \beta \beta$ process, with width representing the systematic uncertainty on the reconstructed energy. Top: normalized residuals between the three component fit and data.

The selected events are fitted around the Q-value of the process, the so called region of interest (ROI), between $[2465,2575] \mathrm{keV}$ (see Fig. 3). A three components simultaneous unbinned extended maximum likelihood (UELM) fit is performed: a flat background from $\alpha$ contributions, a peak accounting for the two coincident $\gamma$ from ${ }^{60} \mathrm{Co}$ and a peak at the Q-value for the $0 v \beta \beta$; the peak is modeled with the detector response function studied using $\gamma$ lines from calibration data. The signal rate best-fit is $\Gamma_{0 v}=\left(-1_{-0.3}^{+0.4}\right.$ (stat.) \pm 0.1 (syst. $\left.)\right) \times 10^{-25} \mathrm{yr}^{-1}$; with zero signal, the background index best-fit is $B I=(0.014 \pm 0.002)$ counts $/(\mathrm{keV} \cdot \mathrm{kg} \cdot \mathrm{yr})$. The result shows that there is no evidence for $0 v \beta \beta$, thus the results from CUORE, CUORE-0 [11] and Cuoricino [16] experiments are combined together and an upper limit on the decay rate of $T_{1 / 2}^{0 v}>1.5 \times 10^{25} \mathrm{yr}$ at $90 \%$ C.L. is obtained. Interpreting the combined half-life limit as a limit on the effective Majorana neutrino mass, within the framework of models where the $0 v \beta \beta$ is mediated by an exchange of a light Majorana neutrino, yields $m_{\beta \beta}<(110-520) \mathrm{meV}$ at $90 \%$ C.L.

\section{Background and $2 v \beta \beta$ half-life}

In comparison to CUORE-0, the CUORE background shows a strong reduction in the $\gamma$-region (i.e. for energies $\lesssim 2615 \mathrm{keV}$ ) as a result of the effort made on the material selection, screening and cleaning, applied also to the parts of the cryostat near the crystals. In the $\alpha$-region the background contribution is almost consistent between the two experiments, since the same cleaning and assembling procedures are applied in both cases for the structures holding the crystals. An excess of surface ${ }^{210} \mathrm{Po}$ events near $5.4 \mathrm{keV}$ is observed and is under investigation, even if this is not influencing the counts in the ROI (estimated contributions are $\lesssim 1 \%$ ).

A Geant 4 Monte-Carlo simulation is performed for the different background components. A total of 60 background sources are considered, with contamination in both the bulk and surfaces of the material (crystals, copper and lead). The contribution of cosmic muons is also taken into account. The simulated background components are used to reconstruct the full background energy spectrum through a Bayesian fit on data exploiting JAGS. The data exploited for the fit are the same that are used for the $0 v \beta \beta$ analysis. A split of the data is performed to take advantage of the sensitivity of the sub-samples to different background features: a split in crystal multiplicity 
enhances the sensitivity to different background contributions; a geometrical split in inner and outer crystal layers allows to better distinguish contaminants from towers and cryostat materials. Priors are assigned to the normalization factors of each background component in the fit, and are obtained from material screening, CUORE-0 background studies and LNGS cosmic flux. The model obtained in this way is able to well reconstruct the main features of the CUORE background.

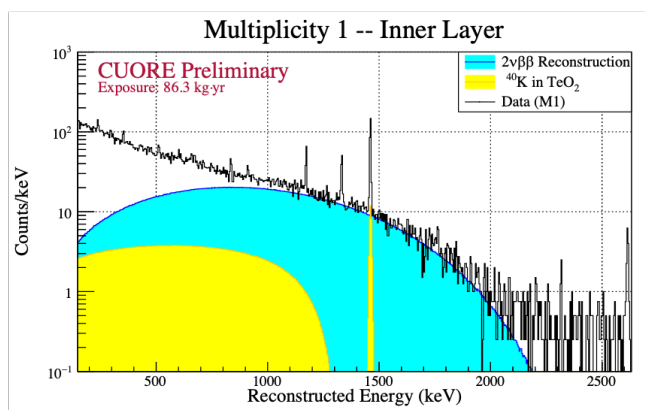

Figure 4: Multiplicity one spectrum in the inner layer (black line) with the reconstructed components of the $2 v \beta \beta$ (blue) and ${ }^{40} \mathrm{~K}$ (yellow) backgrounds.

A side result of the background reconstruction is the measure of the $2 v \beta \beta$ half-life of ${ }^{130} \mathrm{Te}$, since it is one of the components of the background reconstruction. The measured half-life is $T_{1 / 2}^{2 v}=$ $(7.9 \pm 0.1$ (stat. $) \pm 0.2($ syst. $)) \cdot 10^{20} y r$ and is consistent with previous measurements [17, 18]. The reconstructed $2 v \beta \beta$ contribution to the CUORE spectrum, together with the most correlated component from ${ }^{40} \mathrm{~K}$, is shown in Fig. 4. This result represents the most precise measurements of $2 v \beta \beta$ half-life of ${ }^{130} \mathrm{Te}$ to date.

\section{Status of the experiment}

After the first two datasets, CUORE faced three important periods of stops from data-taking for optimization and maintenance purposes: during the first period, a scan of the detector performances versus working temperature has been investigated on physics events from calibration data. Results showed a trend towards better detector resolutions at lower temperatures, thus a new working temperature of $11 \mathrm{mK}$ was chosen for data-taking after 2017. Also, during the first stop a small leak related to the gate valves connecting the detector calibration system (DCS) to the cryostat was fixed; the second and third phases have been devoted to solve small leaks in the cryostat, a partial obstruction of the condensation lines and to fix two PTs. All the periods of stops required to warmup the system to $100 \mathrm{~K}$ to allow the needed interventions. After these periods CUORE is taking data smoothly in stable conditions.

Given its mass and ultra-low background, CUORE is suitable for searches of Dark Matter (DM) induced signals. The capability to search for DM strongly depends on the performances of CUORE in triggering events at very low energies. Simulations show that the expected effects induced by WIMPs extend up to few tens of $\mathrm{keV}$ in the energy spectrum. The trigger thresholds in CUORE on the first two datasets range from $20 \mathrm{keV}$ up to about $100 \mathrm{keV}$. A trigger algorithm based on the Optimal Filter technique has been introduced in CUORE [14] to reduce the current thresholds: the data are filtered in the frequency domain with a filter having a transfer function maximizing the SNR, so the filtered data are less noisy, allowing to achieve lower thresholds. 


\section{Conclusions}

With the data from the first two datasets, corresponding to an exposure of $86.3 \mathrm{~kg} \cdot y \mathrm{r}$, CUORE has been able to set the strongest limit on the $0 v \beta \beta$ half-life of ${ }^{130} \mathrm{Te}$ and to make the most precise measurement of the $2 v \beta \beta$ half-life of ${ }^{130} \mathrm{Te}$. The CUORE Collaboration is also exploring the possibility to lower the trigger energy thresholds by employing a trigger algorithm based on the Optimum Filter technique.

A lot of experience was already gained from the first operations of the CUORE experiment: a noise cancelling method has been developed to reduce the noise induced by the cryogenic system into the detector; refined methods to set the detector working conditions for the $\sim 1000$ crystals have been implemented; studies and tests of new strategies to improve the detector resolution are ongoing.

At present, CUORE has already collected more than $300 \mathrm{~kg} \cdot \mathrm{yr}$ of exposure and keeps running with a high duty cycle and in stable conditions. Updated physics results with new collected data will be released shortly.

\section{References}

[1] C. Arnaboldi et al., [CUORE Collaboration], Nucl. Instrum. Meth. A 518, 775 (2004).

[2] R. Ardito et al., [CUORE Collaboration], hep-ex/0501010.

[3] E. Fiorini and T. O. Niinikoski, Nuclear Instruments and Methods in Physics Research 224, 83 (1984).

[4] C. Enss, D. McCammon, J. Low Temp Phys 151, 5, (2008).

[5] A. D'Addabbo, C. Bucci, L. Canonica, S. Di Domizio, P. Gorla, L. Marini, A. Nucciotti, I. Nutini, C. Rusconi, and B. Welliver, Cryogenics 93, 56-65 (2018).

[6] C. Alduino et al., Cryogenics 102, 9 (2019).

[7] C. Alduino et al. [CUORE Collaboration], JINST 11, P07009 (2016).

[8] C. Alduino et al. [CUORE Collaboration], Phys. Rev. C 97, 055502 (2018).

[9] C. Alduino et al. [CUORE Collaboration], Eur. Phys. J. C 77 (2017).

[10] C. Alduino et al. [CUORE Collaboration], Phys. Rev. C 93, 045503 (2016).

[11] K. ALfonso et al. [CUORE Collaboration], Phys. Rev. Lett. 115, 102502 (2015).

[12] C. Alduino et al. [CUORE Collaboration], Eur. Phys. J. C 77, 543 (2017).

[13] C. Alduino et al. [CUORE Collaboration], Eur. Phys. J. C 77, 532 (2017).

[14] S. Di Domizio, A. Branca, A. Caminata, L. Canonica, S. Copello, A. Giachero, E. Guardincerri, L. Marini, M. Pallavicini and M. Vignati, JINST 13, P12003 (2018).

[15] C. Alduino et al. [CUORE Collaboration], Phys. Rev. Lett. 120, 132501 (2018).

[16] E. Andreotti et al. , Astropart. Phys. 34, 822 (2011).

[17] Alduino,C.etal.(CUORECollaboration),Eur. Phys. J. C77, 13 (2017).

[18] R.Arnoldetal.(NEMO-3Collaboration),Phys. Rev. Lett.107, 062504 (2011). 